\title{
Distribución y variabilidad morfológica de especies de zarzamoras en México (Rubus spp L.)
}

\author{
Distribution and morphological variability of blackberries species in Mexico (Rubus spp L.)
}

\begin{abstract}
Geremias Rodriguez-Bautista', Sergio Damián Segura Ledesma², Serafin Cruz-Izquierdo', José López-Medina³, Alejandra Gutierrez-Espinosa', Nicasio Cruz-Huerta', José Carrillo-Salazar', Luis Manuel Valenzuela Núñez**

1 Colegio de Postgraduados Campus Montecillo. Km 36.5 Carr. México-Texcoco, Texcoco, Edo. de Méx. C.P. 56230.

2 Universidad Autónoma Chapingo. Centro Regional Universitario Centro Occidente. Periférico Independencia Pte. No. 1000 Col. Lomas del Valle, A. P. 8. C. P. 58170. Morelia, Mich.

3 Facultad de Agrobiología "Presidente Juárez". Universidad Michoacana San Nicolás de Hidalgo. Paseo Gral. Lázaro Cárdenas y Berlín S/N, Colonia Viveros. C.P. 60170. Uruapan, Mich.

${ }^{4}$ Facultad de Ciencias Biológicas. Universidad Juárez del Estado de Durango. Av. Universidad S/N. Fracc. Filadelfia. C. P. 35010. Gómez Palacio, Dgo.
\end{abstract}

\section{RESUMEN}

Se evaluaron los factores climáticos determinantes de la distribución de especies de zarzamoras silvestres y la variabilidad morfológica inter e intraespecífica de Rubus adenotrichos Schltdl, R. cymosus Rydb, R. humistratus Steud, $R$. palmeri Rydb, R. pringlei Rydb y R. sapidus Schltdl en Chiapas, Hidalgo y Michoacán, utilizando información ecogeográfica de Global Biodiversity Information Facility. Los datos de pasaporte fueron sometidos al algoritmo Bio-clim/Domain de DIVA-GIS 7.5. Las variables morfológicas se registraron in situ; los factores climáticos se analizaron por componentes principales y las variables morfológicas por correspondencias múltiples. Los resultados muestran que las especies de zarzamora se distribuyen principalmente en las zonas montañosas del Eje Volcánico Transmexicano y la Sierra Madre del Sur. Las especies presentaron variabilidad en los caracteres morfológicos relacionados con tallo, hoja, flor y fruto. Las especies Rubus adenotrichos Schltdl, R. cymosus Rydb y R. pringlei Rydb mostraron variabilidad morfológica entre los sitios de colecta, principalmente en porte de planta, pigmentación del tallo, ápice del foliolo, forma de espinas en tallo, número drupas y polidrupas. La temperatura y precipitación fueron los factores que determinaron la distribución de zarzamoras silvestres. Se presentó variabilidad morfológica entre las especies de zarzamora entre sitios. $R$. pringlei se consideró una frambuesa debido a las características morfológicas de la polidrupa.

Palabras Clave: Rubus spp, recursos fitogenéticos, variabilidad morfológica y zarzamoras silvestres

\section{ABSTRACT}

In this research, climatic elements determining the distribution of six species of wild blackberry inter and intraespecific morphological variability were analyzed. The species studied were Rubus adenotrichus Schltdl, R. cymosus Rydb, R. humistratus Steud, R. palmeri Rydb, R. pringlei Rydb, and R. sapidus Schltdl, from Chiapas, Hidalgo, and Michoacán. Ecogeographical information was obtained from Global Biodiversity Information Facility. Passport data were submitted to Bio-climate algorithm/Domain in DIVAGIS 7.5. Morphological variables were recollected in situ in the states of Chiapas, Hidalgo and Michoacán. Climate data were analyzed by principal components and morphological traits by multiple correspondence. It was found that blackberry species are mainly distributed in mountainous areas in Transmexican Volcanic Belt and Sierra Madre del Sur. Temperature and precipitation were the climatic elements that determined the distribution of wild blackberries in Mexico. The species showed interespecies variability in plant height, stem thorn morphology, stem pilosity, leaf margin, leaf base morphology, and drupes size by polidrupe. Temperature and precipitation determined distribution of wild blackberries. Morphological variability among blackberry species was observed between sites. $R$. pringlei was considered a raspberry due to the morphological characteristics of polyprupe.

Index words: Rubus spp, phytogenetic resources, morphological variability, and wild blackberries.

\section{INTRODUCCIÓN}

El género Rubus spp pertenece a la familia Rosaceae de la que existen alrededor de 250 especies en el mundo (Hao et al, 2015). El subgénero Eubatus pertenece a las zarzamoras (Rubus subgenus Eubatus) y se distribuye en las zonas de alta montaña tropical desde México hasta Ecuador (Cancino-Escalante et al., 2011). En México, de acuerdo con Rzedowski y Calderón de Rzedowski (2005) existen 61 especies de Rubus.

El género Rubus se caracteriza por su variabilidad morfológica (Espinosa et al., 2016), ya que incluye especies arbustivas y leñosas, pequeñas herbáceas trepadoras o postradas, con láminas foliares reducidas (Clark et al., 2007). Las diferencias son propias del género y de sus especies, usualmente las especies de Rubus presentan variabilidad morfológica y genética debido la poliploidía (Glick et al., 2016; Cancino-Escalante et al., 2011), además se presentan hibridación entre sus especies (Šarhanová et al, 2017; Wang 
et al., 2016; Marulanda et al., 2007). Los procesos de selección natural permiten que las zarzamoras se adapten a las condiciones ecológicas de su hábitat y desarrollen mecanismos de resistencia natural a factores abióticos y bióticos (García-Seco et al., 2015; Dujnovic-Purgar et al., 2012).

Moreno et al. (2011), Caetano et al. (2015) y MartínezCruz et al. (2017), sostienen que la colección, la caracterización y la evaluación del germoplasma vegetal es tarea prioritaria para la conservación, mejoramiento y obtención de materiales sobresalientes con características potenciales. La caracterización debe efectuarse en diferentes órganos de la planta, estados fisiológicos y tipos de caracteres, debiéndose analizar en conjunto, otorgándoles la misma importancia (González-Andrés, 2001). La mayoría de los caracteres son heredables; sin embargo, estos son afectados por los factores ambientales (Blum, 2018; Bita y Gerat, 2013; Holeski et al., 2012). Kirchoff y Claßen-Bockhoff (2013) y Santos et al. (2012), señalan que los órganos menos influenciados por los factores ambientales son los más útiles para la descripción morfológica, como flor y fruto; en importancia decreciente las hojas, tronco, ramas, raíces y los tejidos celulares.

En un estudio de seis especies de zarzamora silvestre de Michoacán, México, Segura-Ledesma et al. (2012) reportaron que éstas comparten caracteres morfológicos y distribución eco geográfica. Sin embargo, es importante realizar estudios que permitan ampliar el conocimiento sobre la diversidad genética y morfológica en especies silvestres de zarzamora en diferentes localidades. En el presente estudio se planteó identificar la distribución de las especies Rubus adenotrichos Schltdl, $R$. cymosus Rydb, $R$. humistratus Steud, R. palmeri Rydb, R. pringlei Rydb y R. sapidus Schltdl a partir del Análisis de Información Geográfica (Diva Gis 7.5), conocer los principales factores climáticos que determinan la distribución de las especies de zarzamora silvestre y determinar la variabilidad morfológica inter e intraespecifica de las especies de Rubus adenotrichos Schltdl, R. cymosus Rydb, $R$. humistratus Steud, R. palmeri Rydb, R. pringlei Rydb y R. sapidus Schltdl provenientes de los estados de Chiapas, Hidalgo y Michoacán en México.

\section{MATERIALES Y MÉTODOS}

Se obtuvo información geográfica de seis especies silvestres de zarzamofigra (Rubus adenotrichos Schltdl, R. cymosus Rydb, R. humistratus Steud, R. palmeri Rydb, R. pringlei Rydb y $R$. sapidus Schltdl), con sus respectivos datos de pasaporte ecogeográficos (altitud, longitud y latitud), a partir de la base de datos de Global Biodiversity Information Facilyty (GBIF, 2015) (www.gbif.org). Los datos de pasaporte ecogeográficos de los sitios de colecta se analizaron mediante el algoritmo Bio-clim/Domain de DIVA-GIS versión 7.5 (Hijmans et al., 2004), y se elaboró el mapa de distribución general a través de la metodología de Núñez-Colín y Goytia-Jiménez (2009) y Núñez-Colín (2010). Posteriormente se determinaron los estados con mayor diversidad que abarcaron las seis especies de interés para la caracterización morfológica y con ello reducir los costos de operación.

La caracterización morfológica se llevó a cabo in situ en los estados de Chiapas, Hidalgo y Michoacán (Tabla 1). Las variables morfológicas se registraron con base en descriptores cualitativos (Guías de la UPOV, 2006) y la confirmación de las especies se realizó mediante la revisión de herbario del Instituto de Ecología, A. C., México (IE-BAJIO).

Las escalas que se usaron para registrar las variables fueron: nominal (n), ordinal (o) y binario (b), y se clasificaron de acuerdo con el órgano (tallo, hoja, flor y fruto) de la planta. Las variables de tallo fueron: porte de la planta (PP, n), longitud del tallo principal (TLP, o), diámetro del tallo principal (TDP, o), número de ramas nuevas (TRN, o), longitud de las ramas nuevas (TLR, o), pigmentación del tallo (TPP, 0), distribución de las ramas en el tallo principal (TDR, n), cerosidad

Tabla 1. Localización geográfica de los sitios de colecta de las zarzamoras silvestres en México.

Table 1. Geographical location of collection sites for wild blackberries in Mexico.

\begin{tabular}{|c|c|c|c|c|c|c|}
\hline \multirow[t]{2}{*}{ Especies } & \multicolumn{2}{|c|}{ Chiapas } & \multicolumn{2}{|c|}{ Hidalgo } & \multicolumn{2}{|c|}{ Michoacán } \\
\hline & Latitud & Longitud & Latitud & Longitud & Latitud & Longitud \\
\hline R. adenotrichos Schltdl & $16^{\circ} 30^{\prime} 50^{\prime \prime}$ & $92^{\circ} 29^{\prime} 23^{\prime \prime}$ & $20^{\circ} 11^{\prime} 29^{\prime \prime}$ & $98^{\circ} 09^{\prime} 22$ & $19^{\circ} 37^{\prime} 17.6^{\prime \prime}$ & $100^{\circ} 08^{\prime} 59.3^{\prime \prime}$ \\
\hline R. cymosus Rydb & $16^{\circ} 59^{\prime} 11^{\prime \prime}$ & $92^{\circ} 59^{\prime} 07^{\prime \prime}$ & $20^{\circ} 16^{\prime} 21$ & $98^{\circ} 09^{\prime} 08^{\prime \prime}$ & $19^{\circ} 37^{\prime} 01.3^{\prime \prime}$ & $101^{\circ} 32^{\prime} 54.1^{\prime \prime}$ \\
\hline R. humistratus Steud & ---------------- & -------------- & --------------- & ---------------- & $19^{\circ} 29^{\prime} 25.7^{\prime \prime}$ & $101^{\circ} 32^{\prime} 54.1^{\prime \prime}$ \\
\hline R.palmeri Rydb & --------------- & 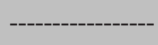 & $20^{\circ} 09^{\prime} 60^{\prime \prime}$ & $98^{\circ} 15^{\prime} 38^{\prime \prime}$ & - & - \\
\hline R. pringlei Rydb & $16^{\circ} 47^{\prime} 08^{\prime \prime}$ & $92^{\circ} 31^{\prime} 05^{\prime \prime}$ & --------------- & ----------------- & $19^{\circ} 29^{\prime} 25.7^{\prime \prime}$ & $101^{\circ} 41^{\prime} 16.4^{\prime \prime}$ \\
\hline R. sapidus Schltdl & - & - & $20^{\circ} 10^{\prime} 09^{\prime \prime}$ & $98^{\circ} 10^{\prime} 19.7^{\prime \prime}$ & $19^{\circ} 38^{\prime} 33.1^{\prime \prime}$ & $101^{\circ} 15^{\prime} 25.2^{\prime \prime}$ \\
\hline
\end{tabular}


en tallos de principal (TCP, b), número de espinas en tallo principal (TPE, o), densidad de tricomas en tallo (TPD, o), forma del tallo (TFT, $n$ ) y forma de la espina en tallo (TFE, $n$ ). Las variables foliares fueron: longitud de la quinta hoja (HLQ, o); número de foliolos (HNF, n), forma del foliolo (TFF, n), ancho de la hoja (HA, o), forma de la hoja (HF, n), forma de la base de la hoja (HFB, n), forma del ápice de la hoja (HFA, n), espinas en la hoja $(\mathrm{HE}, \mathrm{o})$, color del envés de la hoja $(\mathrm{HCE}, \mathrm{n})$ y margen de la hoja $(\mathrm{HM}, \mathrm{n})$. Las variables del órgano floral fueron: diámetro del pétalo (FDP, o), color de pétalos $(F C P, n)$ y forma de pétalo (FFP, n). Las variables del fruto fueron: fructificación lateral $(D F L, o)$, longitud del fruto (DLF, o), ancho del fruto (DAF, o), número de drupas por polidrupa (DND, o) y tamaño de drupas por polidrupa (DTP, o).

En cada sitio de colecta se obtuvieron los siguientes los factores climáticos mediante el programa de Diva Gis 7.5 (Hijmans et al., 2004): temperatura promedio anual (B1), rango medio diurno (B2, temperatura máxima menos temperatura mínima), isotermalidad ( $\left.B 3, B 1 / B 7^{*} 100\right)$, estacionalidad en temperatura (B4), temperatura máxima del período más caliente (B5), temperatura mínima del período más frío (B6), rango anual de temperatura (B7, $B 5-B 6)$, temperatura media en el trimestre más lluvioso (B8), temperatura promedio en el trimestre más seco (B9), temperatura promedio en el trimestre más caluroso (B10), temperatura promedio en el trimestre más frío (B11) precipitación anual (B12), precipitación en el periodo más lluvioso (B13), precipitación en el periodo más seco (B14), estacionalidad de la precipitación (B15), precipitación en el trimestre más lluvioso (B16), precipitación en el trimestre más seco (B17), precipitación en el trimestre más caluroso (B18) y precipitación en el trimestre más frío (B19).

Con los datos de los factores climáticos se realizó un análisis de componentes principales (ACP), mientras que las variables morfológicas se analizaron mediante análisis de correspondencias múltiples (ACM), con el uso del paquete estadístico SAS (Statistical Analysis System Institute Inc.) (SAS, 2002).

\section{RESULTADOS Y DISCUSIÓN Distribución geográfica}

Se encontraron 66 sitios de colecta de la especie Rubus adenotrichus Schltdl, 35 de $R$. cymosus Rydb, $11 R$. humistratus Steud, 11 R. palmeri Rydb, 36 R. pringlei Rydb y 8 de $R$. sapidus Schltdl de la base de datos GBIF. La especie Rubus adenotrichos Schtdl presentó mayor número de sitios distribuidas en el país. Por otro lado, dicha distribución es importante desde el punto de vista nutraceútico, debido que Rubus adenotrichos Schltdl presenta altos contenidos de elagininas (2.2 a 3.8 a mg g-1) y de antocianinas ( 0.20 a 1.34 $\mathrm{mg} \mathrm{g}^{-1}$ ); que favorecen la dieta humana (Acosta-Montoya et al., 2010).

Las especies se distribuyeron principalmente en la región que Morrone (2005) denominó como la región biogeográfica Eje Volcánico Transmexicano y Sierra Madre del Sur. De acuerdo con Ataei-Jaliseh et al. (2015), las especies de zarzamora se distribuyen ampliamente en diferentes hábitats debido a la variabilidad morfológica que presentan de acuerdo a las condiciones ambientales de cada sitio de colecta. Estos resultados contribuyen a planear la colecta de germoplasma con la finalidad de tener la mayor diversidad de especies de interés (Hijmans et al., 2004). Al respecto, Núñez-Colín (2010) menciona que estas regiones son aptas para establecer un banco de germoplasma in situ.

Los resultados muestran que, Rubus cymosus Rydb y Rubus pringlei Rydb no se localizan en Chiapas (Figura 1), sin embargo, durante el muestreo en campo en el estado de Chiapas pudieron encontrarse dichas especies. En contraste, Rubus palmeri Rydb y R. sapidus Schltdl no se encontraron en el lugar debido principalmente a que en los sitios donde fueron reportados en la actualidad se constató que cambió el uso de suelo de forestal a agrícola.

\section{Análisis de los elementos climáticos}

En el análisis de componentes principales, los primeros dos componentes explicaron el $78 \%$ de la variabilidad total y con el tercer componente acumuló el 90 \% (Tabla 2). En el CP1 y CP2, los factores climáticos relacionados con la temperatura y precipitación (temperatura promedio anual, rango medio diurno, estacionalidad en temperatura, temperatura máxima del periodo más caliente, temperatura mínima del periodo más frío, rango anual de temperatura, temperatura media en el trimestre más lluvioso, precipitación anual y precipitación en el trimestre más seco) destacaron para determinar la distribución de las especies silvestres de zarzamora (Figura 2). La temperatura es uno de los factores que presenta impacto en la calidad de los frutos, Granelli et al. (2012), encontraron que en los frutos de zarzamora presentan diferencias en peso del fruto, contenido de sólidos solubles y acidez titulable en función de los cambios de temperatura diurna y nocturna.

Tabla 2. Valores propios y proporción de la varianza explicada por Análisis de Componentes Principales.

Table 2. Own values and variance proportion explained by Principal Component Analysis.

\begin{tabular}{lcccc}
\hline Componente & $\begin{array}{c}\text { Valor propio } \\
(\chi / \mathbf{p})\end{array}$ & Diferencia & $\begin{array}{c}\text { Proporción } \\
(\%)\end{array}$ & $\begin{array}{c}\text { Acumulada } \\
(\%)\end{array}$ \\
\hline CP1 & 8.76 & 2.65 & 0.46 & 0.46 \\
CP2 & 6.12 & 3.80 & 0.32 & 0.78 \\
CP3 & 2.31 & 1.22 & 0.12 & 0.90 \\
CP4 & 1.08 & 0.74 & 0.06 & 0.96 \\
\hline
\end{tabular}

$\mathrm{CP}=$ componente principal.

De acuerdo con la separación angular que forman las proyecciones de las variables (Figura 2), se encontraron asociaciones entre los factores climáticos: temperatura promedio anual con temperatura promedio en el trimestre más seco, precipitación en el trimestre más caluroso con precipitación en el periodo más lluvioso y precipitación en el trimestre más frío; que indican que la precipitación favorece la acumulación de unidades frío de las zarzamoras; CancinoEscalante et al. (2011) mencionan que las asociaciones de las 


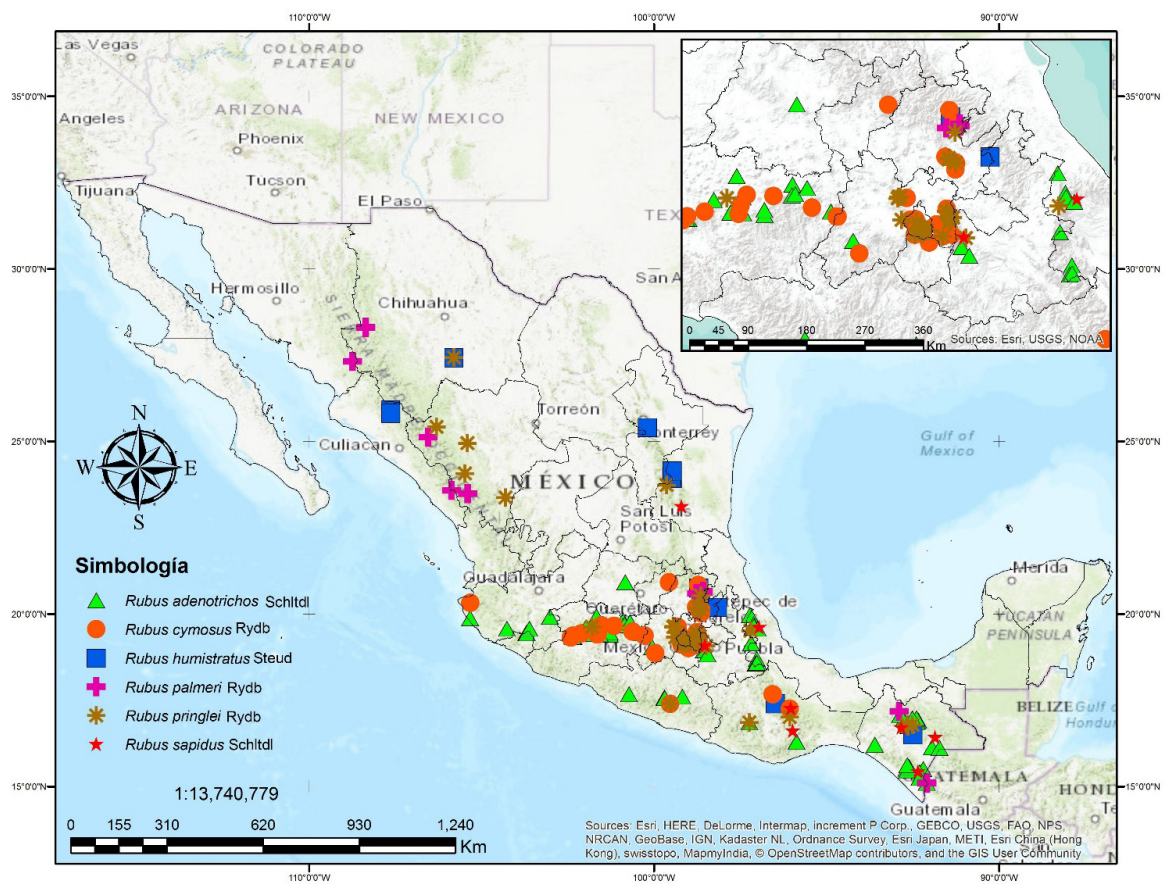

Figura 1. Mapa de distribución de especies silvestres de zarzamora en México, elaborado con información de la Plataforma Mundial de Información en Biodiversidad (GBIF). Elaboró: Geremías Rodríguez Bautista y José Antonio Hernández Herrera.

Figure 1. Distribution map of wild blackberries species in Mexico, done with Global Biodiversity Information Platform (GBIF) information. Done by: Geremías Rodríguez Bautista and José Antonio Hernández Herrera.

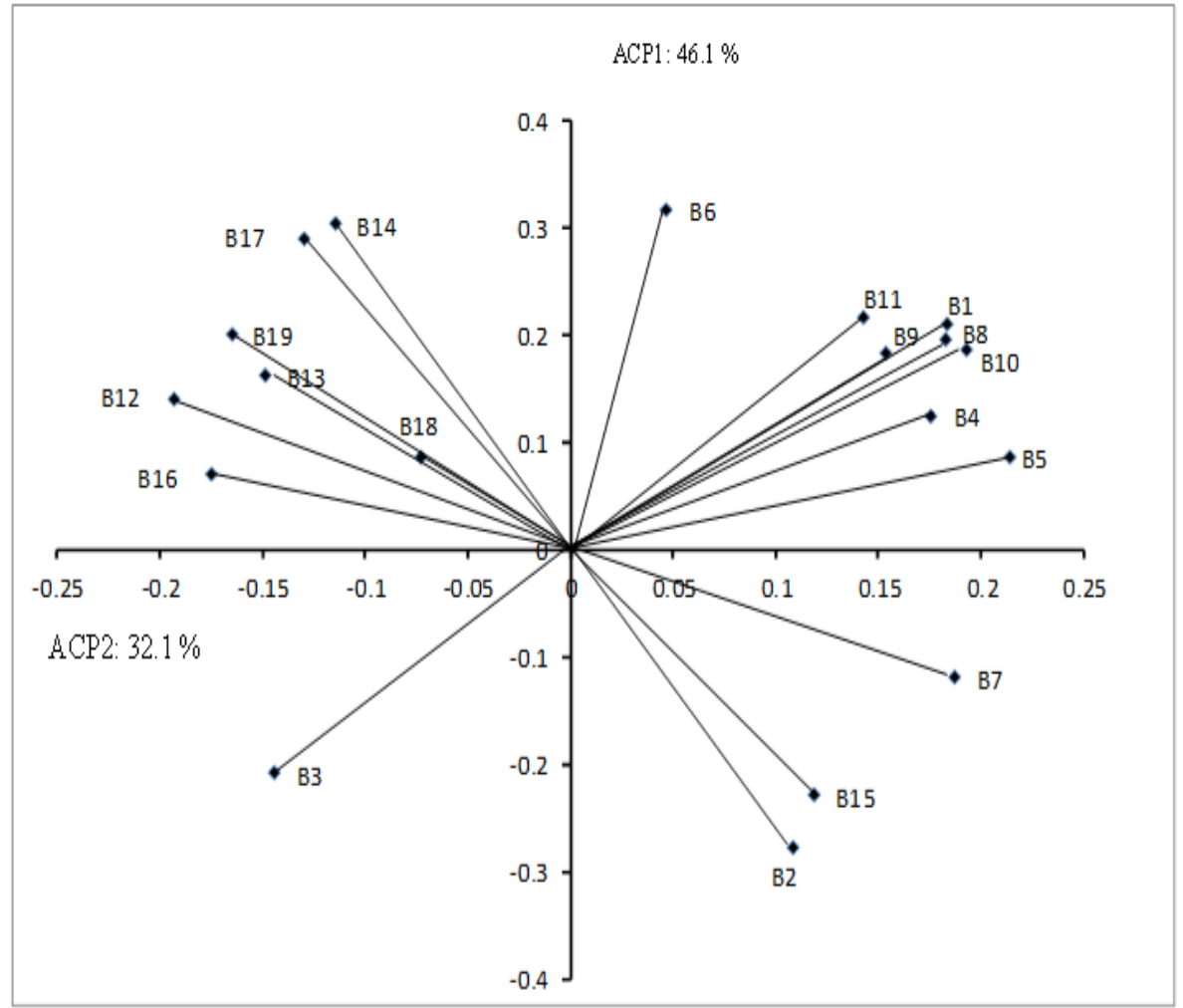

Figura 2. Asociación de los elementos climáticos sobre los dos primeros componentes que caracterizan a las zarzamoras silvestres en México.

Figure 2. Climatic elements association on the first two components characterizing wild blackberries in Mexico. 
variables dependen de la constitución genética de la especie y de la interacción genotipo por ambiente, por lo tanto los individuos expresan su genotipo de acuerdo al hábitat donde se desarrollan.

\section{Variabilidad morfológica inter e intraespecífica}

El análisis de correspondencia múltiple permitió establecer un total de cinco factores que explicaron el 73.1 $\%$ de la variabilidad total, misma que se obtuvo del análisis de variables morfológicas. El comportamiento se explica por la variabilidad morfológica y genética en el género Rubus, en la que se encuentran especies diploides a dodecaploides (Marulanda et al., 2007). Por otro lado, Cancino-Escalante et al. (2011) mencionan que el sistema de reproducción de las plantas influye en la variabilidad, por el efecto de la hibridación entre especies de Rubus.

La dispersión de las especies en el diagrama bidimensional en un plano mostró la variación entre las especies provenientes de los estados de Chiapas, Hidalgo y Michoa- cán (Figura 3). El Factor 1 se definió por las variables número de drupas por polidrupa, porte de la planta y forma de la espina en tallo, mientras que el Factor 2 fue definido por las variables densidad de tricomas en tallo, margen de la hoja y forma de la base de la hoja. Por lo tanto, los resultados que se obtuvieron coinciden con Cancino-Escalante et al. (2011), es decir, los caracteres de tallo, órganos foliares y reproductivos presentaron mayor variabilidad entre especies de zarzamora silvestre. Stephens et al. (2012) encontraron correlación genética entre las variables peso del fruto, longitud y diámetro del tallo. Por lo tanto, es importante incluir variables que involucren los componentes de rendimiento en los estudios de caracterización para definir especies silvestres de mayor rendimiento y calidad de fruto. De acuerdo con Dosett y Finn (2015) la variación fenotípica permite seleccionar las especies silvestres de Rubus para mejoramiento genético. Con la finalidad de incorporar características como vigor y resistencia a enfermedades a partir de especies silvestres.

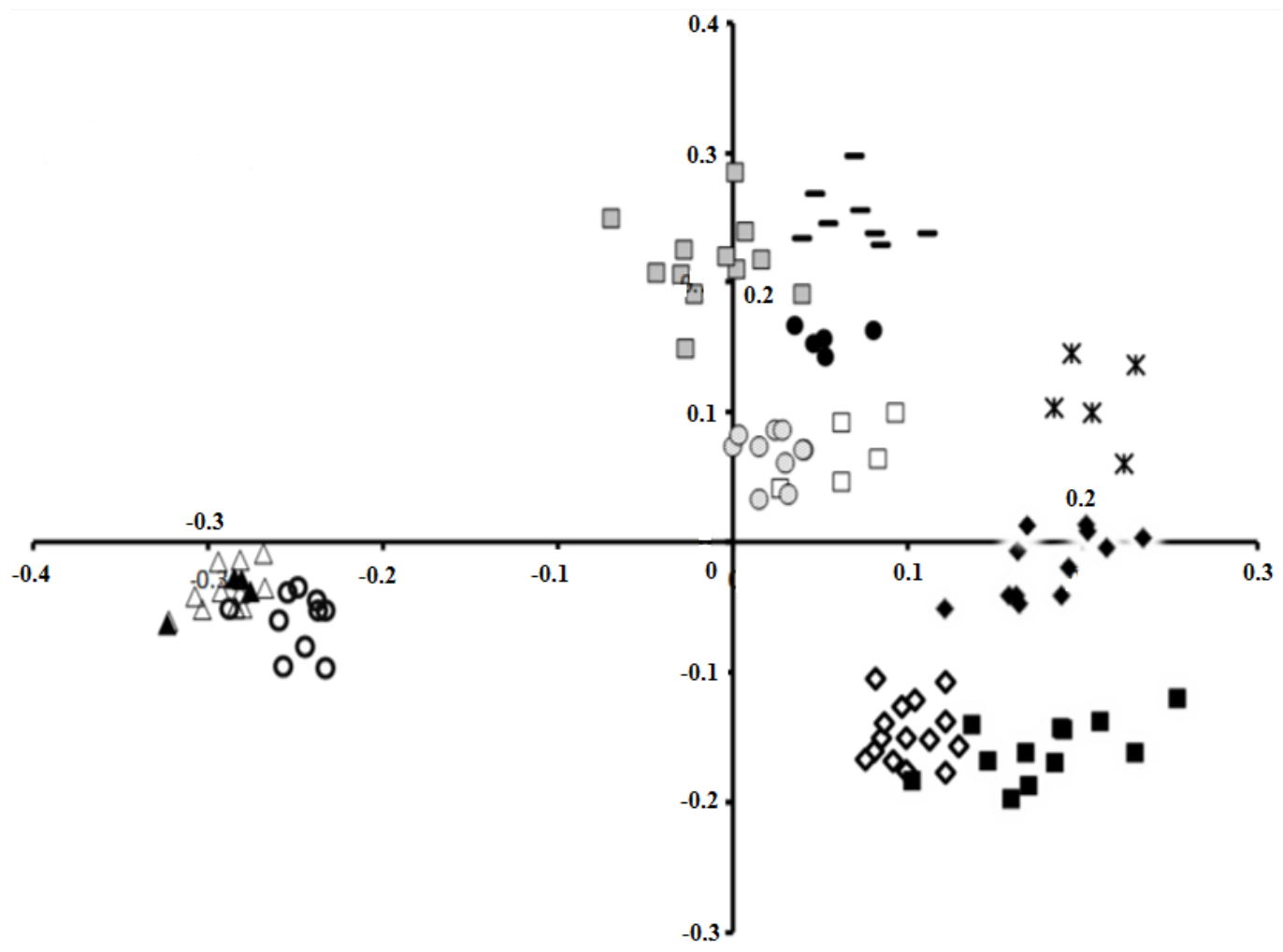

Figura 3. Dispersión de las especies silvestres de zarzamora sobre el plano de los dos primeros factores: Factor 1: 32.1; Factor

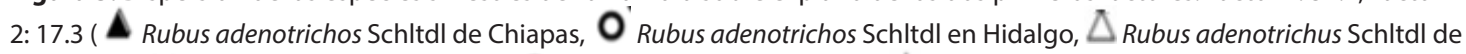

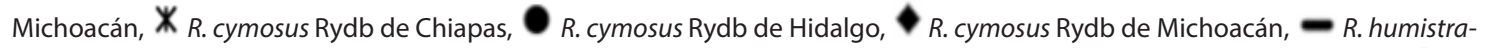

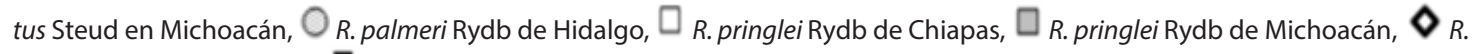

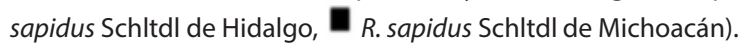

Figure 3. Dispersion of blackberry wild species on the plane of the first two factors: Factor 1: 32.1; Factor 2: 17.3 ( $\boldsymbol{\Delta}_{\text {Rubus ade- }}$ notrichos Schltdl from Chiapas, $\mathbf{O}^{\prime}$ Rubus adenotrichos Schltdl from Hidalgo, $\Delta$ Rubus adenotrichus Schltdl from Michoacán, $\boldsymbol{X}$

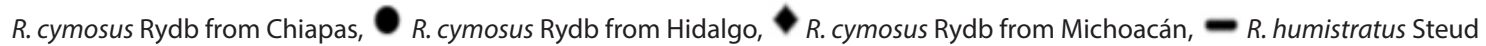

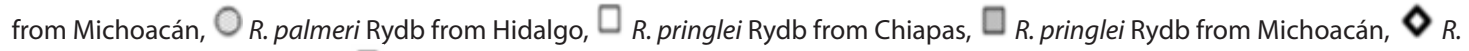

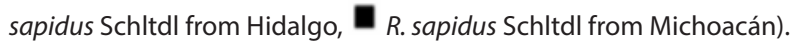


En general las especies de zarzamora silvestre presentaron variación en los caracteres morfológicos tallo, hoja, flor y fruto (Tabla 3). Dichos resultados coinciden con los obtenidos por Ataei-Jaliseh et al. (2015), donde siete especies estudiadas variaron en la densidad de tricomas en tallo. Por otro lado, Monasterio y Macía (2002) reportaron que en las especies de Rubus, la superficie de las distintas partes de la planta está cubierta de tricomas y espinas, cuyo número y forma varían de una especie a otra, modificándose en función de los factores ecológicos y exposición a la radiación solar. Sin embargo, Moreno et al. (2011), mencionan que las espinas son atributos no deseables dentro de los programas de mejoramiento en Rubus, debido que dificultan las labores del cultivo.

En relación al número de foliolos, los resultados concuerdan con los reportados por Cancino-Escalante et al. (2011), quiénes encontraron que $R$. adenotrichos presentó tallos acanalados, foliolos ovados, 3 a 5 foliolos y ápices acuminados (Figura 4) el color y la forma del pétalo permitieron diferenciar las especies (Figura 5) describir el color y las especies. En cuanto a los caracteres del fruto como: número de drupas por polidrupa y número de polidrupas por racimo, $R$. adenotrichos y $R$. pringlei presentaron caracteres reproductivos deseables para un programa de mejoramiento genético (Figura 6), el número de drupas por polidrupa coincide con lo reportado por Zamorano et al. (2007) en materiales comerciales de zarzamora.
En el análisis de la variabilidad morfológica intraespecifica, $R$. sapidus de Hidalgo y Michoacán mostraron agrupación estrecha en función del origen o sitio de estudio, por el contrario, $R$. cymosus presentaron variabilidad entre sitios de colecta. Este comportamiento lo reportó CancinoEscalante et al. (2011) y explican que ningún carácter o grupo de caracteres conforman grupos a los que pertenezcan todos los miembros de la misma especie, aunque provengan de un mismo ambiente, localidad o rango altitudinal.

La especie $R$. cymosus proveniente del estado de Hidalgo y Michoacán presentó porte de planta semierecto y longitud de ramas largas. En contraste, la colecta proveniente de Chiapas fue de porte semierecta a rastrera y longitud de ramas laterales cortas. En relación a la fructificación, en las colectas provenientes de Chiapas y Michoacán, el número de drupas por polidrupa fue medio, es decir, de 9 a 14 y de 8 a 12 , respectivamente, en tanto que, las colectas del estado de Hidalgo presentaron un número bajo, es decir, de 3 a 7.

La especie Rubus pringlei proveniente de Chiapas no presentó pigmentación de antocianinas en tallos; las espinas en tallos y hojas fueron curveadas, y el número de drupas por polidrupa fue alto de 30 a 50 . En contraste, $R$. pringlei proveniente de Michoacán mostró pigmentación con antocianinas en tallos de primocañas y ausentes en floricañas, espinas puntiagudas, y el número de drupas por polidrupa fueron muy alto de 50 a 70 . Estas diferencias morfológicas permitieron agrupar a la especie de $R$. pringlei por

Tabla 3. Características morfológicas interespecificas de las especies silvestres de zarzamora en México.

Table 3. Interspecific morphological characteristics of wild blackberry species in Mexico.

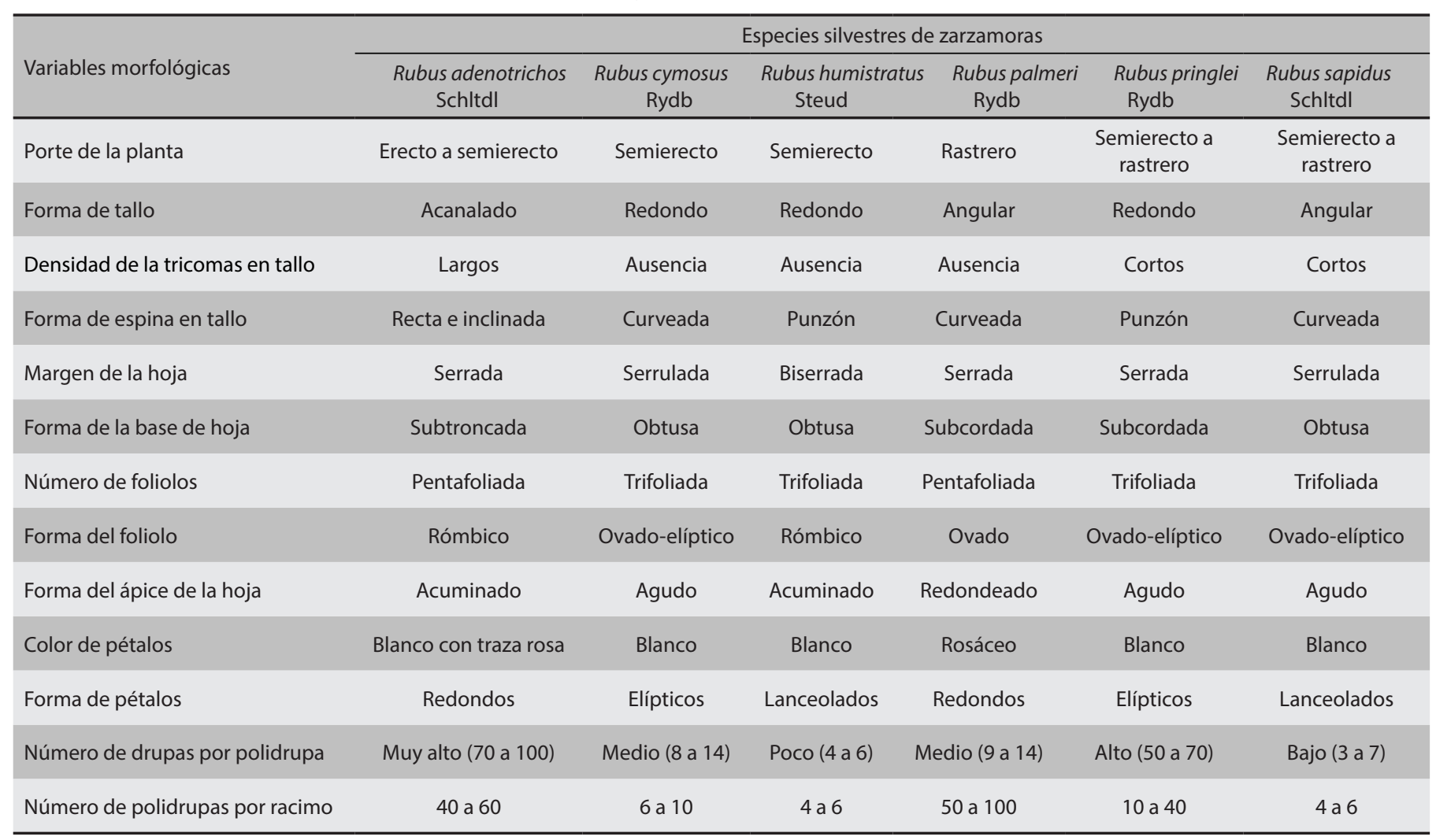




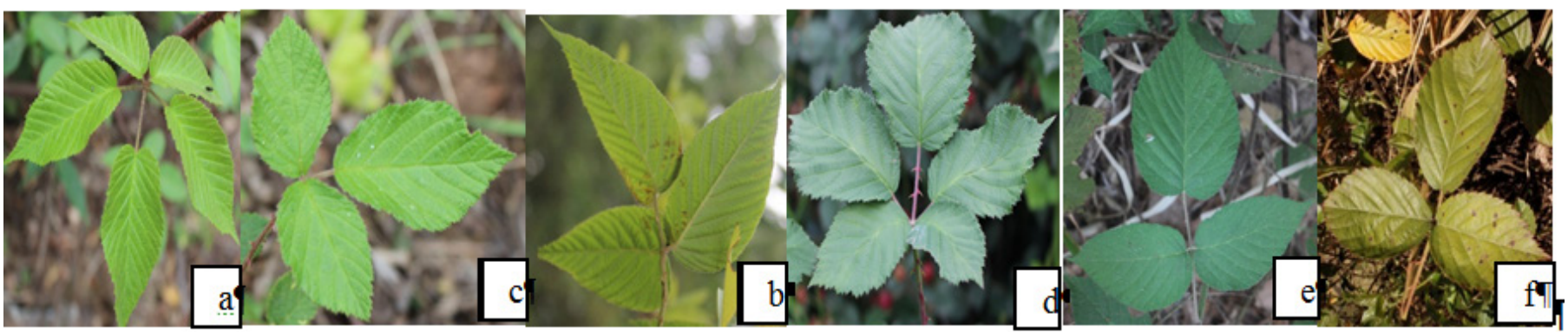

Figura 4. Número de foliolos por hoja: a) Rubus adenotrichos Schltdl, b) R. cymosus Rydb, c) R. humistratus Steud,d) R. palmeri Rydb, e) R. pringlei Rydb y f) R. sapidus Schltdl.

Figure 4. Number of leaflets per leaf: a) Rubus adenotrichos Schltdl, b) R. cymosus Rydb, c) R. humistratus Steud, d) R. palmeri Rydb, e) R. pringlei Rydb and f) R. sapidus Schltdl.

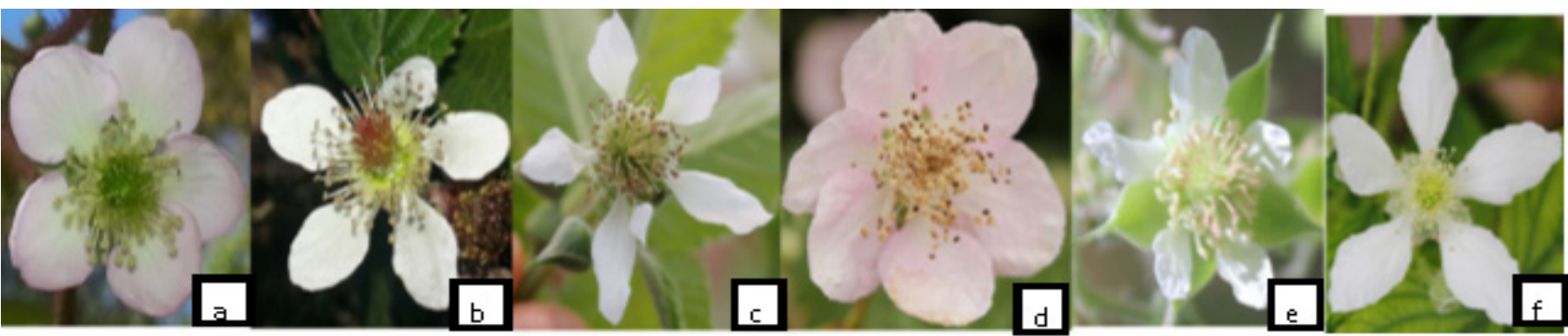

Figura 5. Forma y color de pétalos: a) Rubus adenotrichos Schltdl, b) R. cymosus Rydb, c) R. humistratus Steud, d), R. palmeri Rydb, e) R. pringlei Rydb y f) R. sapidus Schltdl.

Figure 5. Shape and color of petals: a) Rubus adenotrichos Schltdl, b) R. cymosus Rydb, c) R. humistratus Steud, d) R. palmeri Rydb, e) R. pringlei Rydb and f) R. sapidus Schltdl.

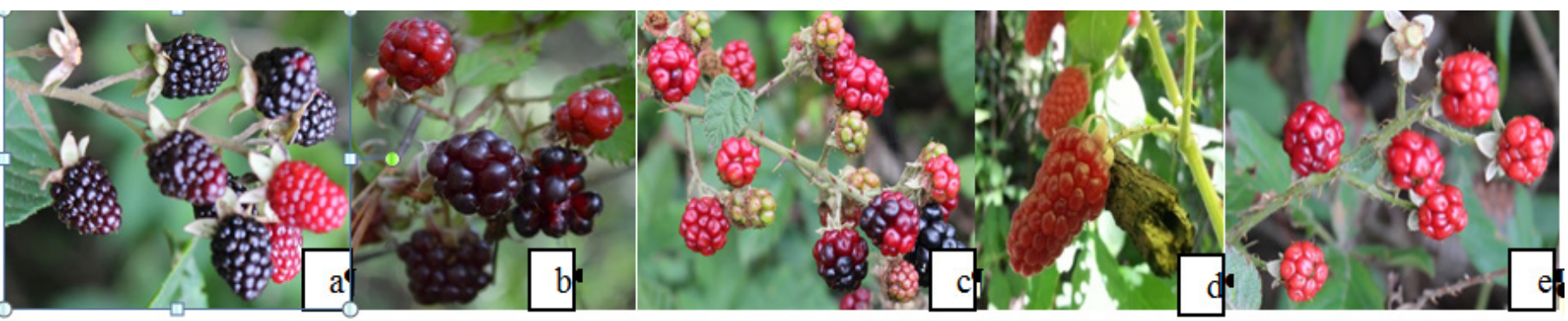

Figura 6. Número de drupas por polidrupa: a) R. adenotrichos Schltdl, b) R. cymosus Rydb, c) R. humistratus Steud, d) R. palmeri Rydb ye) R. pringlei Rydb. Figure 6. Number of drupes per polydrupe: a) R. adenotrichos Schltdl, b) R. cymosus Rydb, c) R. humistratus Steud, d) R. palmeri Rydb, and e) R. pringlei Rydb.

sitio de colecta. Las polidrupas de la especie Rubus pringlei no se adhirieron al receptáculo durante la cosecha, este es un atributo característico de las frambuesas del subgénero Idaeubatus, en contraste a las polidrupas de las zarzamoras se encuentran unidas al receptáculo, mismo que es parte comestible del fruto (Galleta y Violette, 1989), por lo tanto, $R$. pringlei se consideró una frambuesa.

\section{CONCLUSIONES}

Las especies de Rubus adenotrichos, $R$. cymosus, $R$. humistratus, R. palmeri, R. pringlei y R. sapidus se distribuyeron principalmente en el Eje Volcánico Transmexicano y Sierra Madre del Sur. Los factores climáticos relacionados con la temperatura y precipitación determinaron la distribución de las especies de zarzamora. Los caracteres morfológicos relacionados con porte de la planta, forma de la espina en tallo, densidad de tricomas en tallo, margen de la hoja, forma de la base de la hoja y número de drupas por polidrupa presentaron variabilidad morfológica en las especies de zarzamora.
Las especies Rubus adenotrichos, R. cymosus y R. pringlei mostraron variabilidad morfológica intraespecifica entre sitios de colecta. Por las características morfológicas de la polidrupa, R. pringlei se consideró una frambuesa.

\section{AGRADECIMIENTOS}

Al Consejo Nacional de Ciencia y Tecnología (CONACyT) por el financiamiento a la beca otorgada para estudios de Doctorado y al Fideicomiso No.167304 para la Investigación Científica y Desarrollo Tecnológico, por el financiamiento al proyecto "Diversidad genética de poblaciones de zarzamora (Rubus spp)": Se agradece de la misma forma al M. C. José Antonio Hernández Herrera su invaluable apoyo en la elaboración de la cartografía.

\section{REFERENCIAS}

Acosta-Montoya, O., Vaillan, F., Cozzano, S., Mertz, C., Pérez, A.M. y Castro, M.V. 2009 Phenolic content and antioxidant capacity of tropical Highland blackberry (Rubus adenotrichus 
Schltdl) during three edible maturity stages. Food Chemistry 119:1497-1501.

Ataei-Jaliseh, S., Mehregan, I., Tarang, A. y Nejadsattari, T. 2015. A taxonomic review of Rubus L. (Rosaceae) in the Northern Iran based on the analysis of quantitative morphological characters. Journal of Biodiversity and Environmental Sciences 6: 113-120.

Bita, C.E. y Gerats, T. 2013. Plant tolerance to high temperature in a changing environment: scientific fundamentals and production of heat stress-tolerant crops. Frontiers in Plant Science 4:273.

Blum, A. 2018. Plant breeding for stress environments. CRS Press. United States of America. 223 p.

Caetano, C.M., Peña, R.D., Maigual, J.L., Vásquez, L.N., Caetano, D., Pazdiora, B.R. 2015. Mejoramiento participativo: herramienta para la conservación de cultivos subutilizados y olvidados. Acta Agronómica 64 (3): 307- 327.

Cancino-Escalante, G.O., Sánchez-Montaño, L.R., QuevedoGarcía, E. y Díaz-Carvajal, C. 2011. Caracterización fenotípica de accesiones de especies de Rubus $L$. de los municipios de Pamplona y Chitagá, región Nororiental de Colombia. Universitas Scientiarum 16:219-233.

Clark, J.R., Stafne, E.T., Hall, H.K. y Finn, C.E. 2007. Blackberry breeding and genetics. In: Plant Breeding Reviews. Janick J. (ed), John Wiley \& Sons, Inc., New Jersey, Vol. 29. pp:19-144.

Dosett, M. y Finn, C.E. 2015. Performance and phenology of wild black raspberry (Rubus occidentalis L.) germplasm in a common garden. Genetic Resources and Crop Evolution $1-21$.

Dujmović-Purgar, D., Boris, D., Voća, S., Vokurka, A. y Ercisli S. 2012. A comparison of fruit chemical characteristics of two wild grown Rubus species from different locations of Croatia. Molecules 17:10390-10398.

Espinosa B., N., Ligarreto M., G.A., Barrero M., L.S. y Medina C., C.I. 2016. Variabilidad morfológica de variedades nativas de mora (Rubus sp.) en los Andes de Colombia. Revista Colombiana de Ciencias Hortícolas 10 (2) 211:221.

Galleta, G., y Violette C. 1989 The Brambles. In: Brambles Production Guide. M Pritts, D Handley (eds). Northeast Regional Agricultural Engineering Service. Ithaca, New York, E.U. pp: 3-8.

Garcia-Seco, D., Zhang, Y., Gutierrez-Mañero, F.J., Martin, C. y Ramos-Solano, B. 2015. Application of Pseudomonas fluorescens to blackberry under field conditions improves fruit quality by modifying flavonoid metabolism. PLoS ONE 10(11): e0142639.

Global Biodiversity Information Facility (GBIF) (www.gbif.org), Consultado, marzo 2015.

González-Andrés, F. 2001. Caracterización morfológica: In: conservación y Caracterización de Recursos Fitogenéticos. F. González-Andrés y J. Pita-Villamil (eds). Publicaciones Instituto Nacional de Educación Agrícola. Valladolid, España. pp:199-217.

Glick, L., Sabath, N., Ashman, T.L., Goldberg, E. y Mayrose, I. 2016. Polyploidy and sexual system in angiosperms: Is there an association? American Jounal of Botany 103 (7): 1223 - 1235

Granelli G., L. Mariani, S., Parisi, T., Eccher, V., Ughini, R., Lo Scalzo, M., Buccheriy Cortellino G. 2012. Influence of genotype, location and year factor quality and health promoting compunds of Rubus fruits. Acta Horticulturae 926:697-704.
Hao, D.A., Gu, X.J. y Xiao, P.G. 2015. Potentilla and Rubus medicinal plants: potential non-Camellia tea resources. Medicinal Plants, Chemistry, Biology and Omics. 373-430.

Hijmans R., J., Guarino, L., Bussink, C., Mathur, P., Cruz, M., Barrantes, I. y Rojas E. 2004. DIVA-GIS Versión 5.2. Sistema de Información Geográfica para el Análisis de Datos de Distribución de Especies, Guía de Usuario. Centro Internacional de la Papa, Lima, Perú. 83 p.

Holeski LM, Jander Anurag G, Agrawal A. 2012 Transgenerational defense induction and epigenetic inheritance in plants. Trends in Ecology \& Evolution 27(11): 618-626.

Kirchoff, B.K. y Claßen-Bockhoff, R. 2013. Inflorescences: concepts, function, development and evolution. Annals of Botany 112(8): 1471-1476.

Martínez-Cruz, M., Ríos-Labrada, H., Ortiz Pérez, R., MirandaLorigados, S., Acosta-Roca, R., Moreno-Moreno, I., PonceBrito, M., De-la-Fé-Montenegro, C.F., Martin, L. 2017. Metodología del Fitomejoramiento Participativo (FP) en Cuba. Cultivos Tropicales 38 (4): 132-138.

Marulanda, M.L., López, A.M. y Aguilar, S.B. 2007. Genetic diversity of wild and cultivated Rubus species in Colombia using AFLP and SSR markers. Crop Breeding and Applied Biotechnology 7:242-252.

Monasterio, H. y Macía, E. 2002. Revisión taxonómica del género Rubus $L$. (Rosácea) en la Península Ibérica e Islas Baleares. Tesis Doctoral. Departamento de Biología Vegetal Facultad de Farmacia. Universidad Complutense de Madrid, Madrid. $217 \mathrm{p}$.

Moreno M., D., Villarreal, T.C., Lagos, Ordoñez, H. y Criollo, H. 2011. Caracterización "In situ" de genotipos silvestres y cultivadas de mora Rubus spp en el municipio de Pasto. Revista de Ciencias Agrícolas 27:109-128.

Morrone, J.J. 2005. Hacia una síntesis biogeográfica de México. Revista Mexicana de Biodiversidad 76:207-252.

Núñez-Colín, C.A. 2010. Distribución y caracterización ecoclimática del membrillo cimarrón (Amelanchier denticulata (Kunth) Koch) en México. Revista Chapingo Serie Horticultura 16:195-206.

Núñez-Colín, C.A. y Goytia-Jiménez, M.A. 2009. Distribution and agroclimatic characterization of potential cultivation regions of physic nut in México. Pesquisa Agropecuaria Brasilia 44: 1078-1085.

Rzedowski, J. y Calderón-de-Rzedowski, G. 2005. Flora del Bajío y de Regiones Adyacentes, Familia Rosácea. Instituto de Ecología A. C. Centro Regional del Bajío Pátzcuaro, Morelia, Michoacán. No. 135. 163 p.

Santos, R.C., Pires, J.L. y Correa, R.X. 2012. Morphological characterization of leaf, flower, fruit and seed traits among Brazilian Theobroma L. species. Genetic Resources Crop Evolution 59:327-345.

Šarhanová P, Sharbel TF, Sochor M, Vašut RJ, Dančák $M$, Trávníček B. 2017. Hybridization drives evolution of apomicts in Rubus subgenus Rubus: evidence from microsatellite markers. Annals of Botany 120 (2): 317-328.

Statistical Analysis System (SAS). 2002. SAS System for Windows Version 8.0 by SAS Institute Inc. Cary, NC. USA.

Segura-Ledesma, S., Rebollar-Alviter, Á., Boyzo-Marín, J., Hernández-Bello, M. y López-Medina, J. 2012. Genetic Resources of Blackberry Wild Species in Michoacán, México. Acta Horticulturae 946:107-111. 
Stephens, M.J., Alspach, P.A. y Winefield, C. 2012. Genetic parameters associated con yield and yield components in red raspberry. Acta Horticulturae 946:37-42.

Unión Internacional para la Protección de las obtenciones Vegetales (UPOV). 2006. Rubus subgenus Eubatus sect. Moriferi \& Ursini e híbridos (www.upov.int), consultada, octubre 2014.
Wang, Nan YH, Chen Q, He W, Zhang L, Wang XR. Tang HR. 2016. Genomic in situ hybridization analysis between Rubus coreanus and its relatives in Rubus (Sect. Idaeobatus). Plant Biosystems - An International Journal Dealing with all Aspects of Plant Biology 150 (3): 404-411.

Zamorano, M., Morillo A.C., Morillo, Y.C., Vásquez, H.A., y Muñoz, J.E.F. 2007. Caracterización morfológica de mora en los departamentos del Valle del Cauca, Cauca y Nariño, de Colombia. Acta Agronómica 56:51-60. 